\title{
Finding Top-k Profitable Products
}

\author{
Qian Wan, Raymond Chi-Wing Wong, Yu Peng \\ Computer Science and Engineering Department, Hong Kong University of Science and Technology \\ Clear Water Bay, Kowloon, Hong Kong \\ \{qwan, raywong, gracepy\}@cse.ust.hk
}

\begin{abstract}
The importance of dominance and skyline analysis has been well recognized in multi-criteria decision making applications. Most previous studies focus on how to help customers find a set of "best" possible products from a pool of given products. In this paper, we identify an interesting problem, finding top- $k$ profitable products, which has not been studied before. Given a set of products in the existing market, we want to find a set of $k$ "best" possible products such that these new products are not dominated by the products in the existing market. In this problem, we need to set the prices of these products such that the total profit is maximized. We refer such products as top- $k$ profitable products. A straightforward solution is to enumerate all possible subsets of size $k$ and find the subset which gives the greatest profit. However, there are an exponential number of possible subsets. In this paper, we propose solutions to find the top- $k$ profitable products efficiently. An extensive performance study using both synthetic and real datasets is reported to verify its effectiveness and efficiency.
\end{abstract}

\section{INTRODUCTION}

Dominance analysis is important in many multi-criteria decision making applications.

Example 1 (Skyline): Consider that a customer is looking for a vacation package to Hannover using some travel agencies like Expedia.com and Priceline.com. The customer uses two criteria for choosing a package, namely price and distance-to-beach, where price is the price of a package and distance-to-beach is the distance between a hotel in a package and a beach. For two packages $p$ and $q$, if $p$ is better than $q$ in at least one factor, and is not worse than $q$ in any other factors, then $p$ is said to dominate $q$. Table I shows four packages: $p_{1}, p_{2}, p_{3}$ and $p_{4}$. We know that lower price and shorter distance-to-beach are more preferable. Thus, $p_{3}$ dominates $p_{4}$ because $p_{3}$ has lower price and shorter distanceto-beach than $p_{4}$. However, $p_{3}$ does not dominate $p_{1}$ because $p_{1}$ has lower price than $p_{3}$. Similarly, $p_{1}$ does not dominate $p_{3}$ because $p_{3}$ has shorter distance-to-beach.

A package which is not dominated by any other packages is said to be a skyline package or it is in the skyline. Recently, skyline analysis has received a lot of interest in the literature [10], [6], [14], [5], [9], [15]. The packages in the skyline are the best possible tradeoffs among the two factors in question. In Example 1, $p_{3}$ is in the skyline because it is not dominated by $p_{1}, p_{2}$ and $p_{4}$. However, $p_{4}$ is not in the skyline because it is dominated by $p_{3}$.

Consider that a new travel agency wants to start some new packages from a pool of potential packages as shown in Table II. Table II shows three potential packages, namely $q_{1}, q_{2}$

\begin{tabular}{|c|c|c|}
\hline Package & Distance-to-beach & Price \\
\hline$p_{1}$ & 7.0 & 200 \\
\hline$p_{2}$ & 4.0 & 350 \\
\hline$p_{3}$ & 1.0 & 500 \\
\hline$p_{4}$ & 3.0 & 600 \\
\hline
\end{tabular}

TABLE I

PACKAGES IN THE EXISTING MARKET

\begin{tabular}{|c|c|c|c|}
\hline Package & Distance-to-beach & Price & Cost \\
\hline$q_{1}$ & 5.0 & $?$ & 100 \\
\hline$q_{2}$ & 4.5 & $?$ & 200 \\
\hline$q_{3}$ & 0.5 & $?$ & 400 \\
\hline
\end{tabular}

TABLE II

Potential PACKAGES IN THE NEW TRAVEL AGENCY

and $q_{3}$. In this table, attribute distance-to-beach and attribute cost of each package are given. However, attribute price is to be determined by the agency.

Example 2 (Profitable Price with One Package): Suppose that we select only one new package, says $q_{1}$. What price should we set for package $q_{1}$ ? If we set the price of $q_{1}$ to be $\$ 100$, since the cost of $q_{1}$ is $\$ 100$, the profit of $q_{1}$ is equal to $\$ 100-\$ 100=\$ 0$. In other words, we cannot earn any profit. If we set the price to be $\$ 400$, although we can earn $\$ 400-\$ 100=\$ 300$, this new package $q_{1}$ is dominated by $p_{2}$ in the existing market. In other words, it is likely that no customer will select $q_{1}$ since $p_{2}$ is better than $q_{1}$. However, if we set the price to be $\$ 300$, not only can we earn $\$ 300-\$ 100$ $=\$ 200$ but also $q_{1}$ is not dominated by any packages in the existing market. We say that $\$ 300$ is a profitable price of $q_{1}$ but $\$ 100$ and $\$ 400$ are not profitable prices of $q_{1}$.

Let us consider another example that we want to select only one new package $q_{2}$ (instead of $q_{1}$ ). Similarly, if we set the price to be $\$ 200$, the profit is $\$ 0$. If we set the price to be $\$ 400$, $q_{2}$ is dominated by $p_{2}$. However, if we the price to be $\$ 300$, we can earn $\$ 100$ and $q_{2}$ is not dominated by any packages in the existing market. Thus, $\$ 300$ is a profitable price of $q_{2}$ but $\$ 200$ and $\$ 400$ are not.

Unfortunately, how we set the price of a new package may affect how we set the price of another new package.

Example 3 (Profitable Price with Two Packages): Suppose that we are interested in selecting two new packages, says $q_{1}$ and $q_{2}$, instead of only one new package. From Example 2, if we set both the price of $q_{1}$ and the price of $q_{2}$ to be $\$ 300$ separately, then we can earn some profits and they 
are not dominated by any packages in the existing market. However, after we set these prices, the new package $q_{1}$ is dominated by another new package $q_{2}$. An alternative price setting/assignment is that the price of $q_{1}$ is set to $\$ 250$ and the price of $q_{2}$ is set to $\$ 300$. In this assignment, it is easy to verify that $q_{1}\left(q_{2}\right)$ is not dominated by not only any packages in the existing market but also another new package $q_{2}\left(q_{1}\right)$. Besides, the profit of $q_{1}$ and the profit of $q_{2}$ are $\$ 150$ and $\$ 100$, respectively. The sum of these profits is equal to $\$ 250$.

In this paper, we study the following problem: Given a set $P$ of packages in the existing market and a set $Q$ of potential new packages, we want to select $k$ packages from $Q$ such that the sum of the profits of the selected packages is maximized and each selected package is not dominated by any packages in the existing market and any selected new packages where $k$ is a positive integer and is a user parameter. We call this problem finding top- $k$ profitable products $(T P P)$. For example, $k$ is equal to 1 in Example 2 while $k$ is equal to 2 in Example 3.

A naive way for this problem is to enumerate all possible subsets of size $k$ from $Q$, calculate the sum of the profits of each possible subset, and choose the subset with the greatest sum. However, this approach is not scalable because there are an exponential number of all possible subsets. This motivates us to propose efficient algorithms for problem TPP.

Although how we set the price of a new package may affect how we set the price of another new package and there are an exponential number of possible subsets, interestingly, we propose a dynamic programming approach which finds an optimal solution when there are two attributes to be considered. However, we show that this problem is NP-hard when there are more than two attributes to be considered. Thus, we propose two greedy algorithms for this problem. One greedy algorithm has a theoretical guarantee on the profit returned while the other greedy algorithm performs well empirically.

Finding top- $k$ profitable products is common in many real life applications. Other applications include finding profitable laptops in a new laptop company, finding profitable delivery services in a new cargo delivery company and finding profitable e-advertisements in a webpage.

Our contribution are summarized as follows.

1) To the best of our knowledge, we are the first to study how to find top- $k$ profitable products. Finding top- $k$ profitable products can help the effort of companies to find a subset of products together with their corresponding profitable prices, which cannot be addressed by existing methods.

2) We propose a dynamic programming approach which can find an optimal solution when there are two attributes to be considered. We show that this problem is NP-hard when there are more than two attributes. Thus, we propose two greedy approaches to solve the problem efficiently.

3) We present a systematic performance study using both real and synthetic datasets to verify the effectiveness and the efficiency of our proposed approaches. The experimental results show that finding top- $k$ profitable products is interesting.

The rest of the paper is organized as follows. In Section II, we formally define our problem. In Section III, we describe an algorithm for finding the sum of the profits given a set of $k$ selected packages. The dynamic programming approach is developed in Section IV while the greedy approaches are presented in Section V. In Section VI, we give some discussions of the proposed approaches. A systematic performance study is reported in Section VII. In Section VIII, we describe some related work. The paper is concluded in Section IX.

\section{PRoblem Definition}

We first describe the background about skyline in Section IIA. Then, we give some notations used in our problem in Section II-B and our problem in Section II-C.

\section{A. Background: Skyline}

A skyline analysis involves multiple attributes. The values in each attribute can be modeled by a partial order on the attribute. A partial order $\preceq$ is a reflexive, asymmetric and transitive relation. A partial order is also a total order if for any two values $u$ and $v$ in the domain, either $u \preceq v$ or $v \preceq u$. We write $u \prec v$ if $u \preceq v$ and $u \neq v$.

By default, we consider tuples in an $w$-dimensional ${ }^{1}$ space $\mathbb{S}=x_{1} \times \cdots \times x_{w}$. For each dimension $x_{i}$, we assume that there is a partial or total order. For a tuple $p, p . x_{i}$ is the projection on dimension $x_{i}$. For dimension $x_{i}$, if $p . x_{i} \preceq q . x_{i}$, we also simply write $p \preceq_{x_{i}} q$. We can omit $x_{i}$ if it is clear from the context.

For tuples $p$ and $q, p$ dominates $q$ with respect to $\mathbb{S}$, denoted by $p \prec q$, if for any dimension $x_{i} \in \mathbb{S}, p \preceq x_{i} q$, and there exists a dimension $x_{i_{0}} \in \mathbb{S}$ such that $p \prec x_{x_{0}} q$. If $p$ dominates $q$, then $p$ is more preferable than $q$.

Definition 1 (Skyline): Given a dataset $\mathcal{D}$ containing tuples in space $\mathbb{S}$, a tuple $p \in \mathcal{D}$ is in the skyline of $\mathcal{D}$ (i.e., a skyline tuple in $\mathcal{D}$ ) if $p$ is not dominated by any tuples in $\mathcal{D}$. The skyline of $\mathcal{D}$, denoted by $S K Y(\mathcal{D})$, is the set of skyline tuples in $\mathcal{D}$.

For example, in Table I where $\mathcal{D}=\left\{p_{1}, p_{2}, p_{3}, p_{4}\right\}$, since $p_{1}, p_{2}$ and $p_{3}$ are not dominated by any tuples in $\mathcal{D}, S K Y(\mathcal{D})$ is equal to $\left\{p_{1}, p_{2}, p_{3}\right\}$.

\section{B. Notations}

We have a set $P$ of $m$ tuples in the existing market, namely $p_{1}, p_{2}, \ldots, p_{m}$. Each tuple $p$ has $l$ attributes, namely $A_{1}, A_{2}, \ldots, A_{l}$. The domain of each attribute is $\mathbb{R}$. The value of attribute $A_{j}$ for tuple $p$ is given and is denoted by $p . A_{j}$ where $j \in[1, l]$. In particular, the last attribute $A_{l}$ represents attribute price and all other attributes represent the attributes other than price. Besides, we have a set $Q$ of $n$ potential new tuples, namely $q_{1}, q_{2}, \ldots, q_{n}$. Similarly, each tuple $q$ has the

\footnotetext{
${ }^{1}$ In this paper, we use the terms "attribute" and "dimension" interchangeably.
} 
same $l$ attributes, namely $A_{1}, A_{2}, \ldots, A_{l}$. The value of attribute $A_{j}$ for tuple $q$ is denoted by $p . A_{j}$ where $j \in[1, l]$. However, the value of attribute $A_{l}$ for tuple $q$ is not given and the value of each of the other attributes is given. We assume that no two potential new tuples in $Q$ are identical (i.e., no two tuples in $Q$ have the same attribute values for $\left.A_{1}, A_{2}, \ldots, A_{l-1}\right)$. In addition to these $l$ attributes, each tuple $q$ is associated with one additional cost attribute $C$. The value of attribute $C$ for $q$ is denoted by q.C. We assume that for any two tuples in $P \cup Q$, they have at least one attribute value different among the first $l-1$ attributes. This assumption allows us to avoid several complicated, yet uninteresting, "boundary" cases. If this assumption does not hold, the proposed algorithms can be modified accordingly.

In our running example, $P$ contains 4 tuples, namely $p_{1}, p_{2}, p_{3}$ and $p_{4}$ (Table I), and $Q$ contains 3 tuples, namely $q_{1}, q_{2}$ and $q_{3}$ (Table II). Attribute $A_{1}$ and attribute $A_{2}$ are "Distance-to-beach" and "Price", respectively. Attribute $C$ is "Cost".

Since there are an infinite number of possible values in $\mathbb{R}$, we assume the domain of attribute "Price" (i.e., $A_{l}$ ) is defined to be $D=\{0, \sigma, 2 \sigma, 3 \sigma, \ldots\}$ where $\sigma$ is a real number and a user parameter. If we want to have a finer granularity, we should set $\sigma$ to a smaller value. This assumption makes sense in real life applications where attribute Price involves discrete values instead of continuous real values. For example, attribute Price has value " $\$ 500.00$ " and value " $\$ 1000.00$ " but no value " $\$ 1000.0007$ ". In the following, we set $\sigma=50$ for our running example.

\section{Finding Top-k Profitable Products}

A new company is interested in selecting $k$ tuples from $Q$ as the final tuples where $k$ is a positive integer and a user parameter. It wants to maximize the profit of this selection. There are many possible subsets containing $k$ tuples from $Q$. Let us consider one particular subset $Q^{\prime}$. The price of each of these $k$ tuples (represented by attribute $A_{l}$ ) in $Q^{\prime}$ is to be assigned with a value in $D$. Given a tuple $q$ in $Q$, after we set $q . A_{l}$ to a value $v$, the profit of $q$, denoted by $\triangle(q, v)$, is defined to be

$$
v-q \cdot C
$$

In our running example, if we set $q_{1} . A_{l}$ to be $\$ 300$, then $\triangle\left(q_{1}, 300\right)$ is equal to $\$ 300-\$ 100=\$ 200$. If we set it to be $\$ 250$, then $\triangle\left(q_{1}, 250\right)$ is equal to $\$ 250-\$ 100=\$ 150$.

We define a price assignment vector of $Q^{\prime}$, denoted by $\mathbf{v}$, in form of $\left(v_{1}, v_{2}, \ldots, v_{n}\right) . v_{i}$ is said to be the $i$-th entry of $\mathbf{v}$. If $q_{i} \in Q^{\prime}$ where $i \in[1, n]$, then $v_{i}$ is assigned with a value in $D$. Otherwise, $v_{i}$ is set to 0 .

Suppose that $q$ is selected in the final selection set $Q^{\prime}$. Our objective is to make sure that after we set the price of $q, q$ is not dominated by not only the tuples in the existing market $(P)$ but also the newly selected tuples in $Q^{\prime}$. That is, $q$ should be in the skyline with respect to $P \cup Q^{\prime}$.

A price assignment vector $\mathbf{v}$ is said to be feasible if after we set the price of each $q_{i} \in Q^{\prime}$ to $v_{i}$, each $q_{i} \in Q^{\prime}$ is in the skyline with respect to $P \cup Q^{\prime}$.
Example 4 (Feasible Vector): In Example 3, $k=2$. Suppose that $Q^{\prime}=\left\{q_{1}, q_{2}\right\}$.

Consider that we set the price of $q_{1}$ to $\$ 300$ and the price of $q_{2}$ to $\$ 300$. The price assignment vector $\mathbf{v}$ is $(300,300,0)$. $\mathbf{v}$ is not feasible. As described in Example 3, after we set the price for $q_{1}$ and the price for $q_{2}, q_{1}$ is not in the skyline with respect to $P \cup Q^{\prime}$ because $q_{1}$ is dominated by $q_{2}$.

Consider that we set the price of $q_{1}$ to $\$ 250$ (instead of $\$ 300$ ) and the price of $q_{2}$ to $\$ 300$. The price assignment vector $\mathbf{v}^{\prime}$ is $(250,300,0) . \mathbf{v}^{\prime}$ is feasible because after we set the price for $q_{1}$ and the price for $q_{2}$, both $q_{1}$ and $q_{2}$ are in the skyline with respect to $P \cup Q^{\prime}$.

Definition 2 (Profit of Selection): Let $Q^{\prime}$ be a set of $k$ tuples selected from $Q$. Let $\mathbf{v}$ be the price assignment vector of $Q^{\prime}$ in form of $\left(v_{1}, v_{2}, \ldots, v_{n}\right)$. The profit of $Q^{\prime}$ with $\mathbf{v}$, denoted by $\operatorname{Profit}\left(Q^{\prime}, \mathbf{v}\right)$, is defined to be

$$
\sum_{q_{i} \in Q^{\prime}} \triangle\left(q_{i}, v_{i}\right)
$$

Example 5 (Profit of Selection): In Example 3, $k=2$. Suppose that $Q^{\prime}=\left\{q_{1}, q_{2}\right\}$.

Consider that the selection set is $Q^{\prime}$. We set the price of $q_{1}$ to $\$ 250$ and the price of $q_{2}$ to $\$ 300$. The price assignment vector $\mathbf{v}^{\prime}$ is $(250,300,0)$. From Example 4, we know that this vector is feasible.

$\operatorname{Profit}\left(Q^{\prime}, \mathbf{v}^{\prime}\right)$ is equal to

$$
\begin{aligned}
& \triangle\left(q_{1}, v_{1}\right)+\triangle\left(q_{2}, v_{2}\right) \\
= & (\$ 250-\$ 100)+(\$ 300-\$ 200) \\
= & \$ 150+\$ 100 \\
= & \$ 250
\end{aligned}
$$

Consider that the selection set is still $Q^{\prime}$. We set the price of $q_{1}$ to $\$ 150$ (instead of $\$ 250$ ) and the price of $q_{2}$ to $\$ 300$. The price assignment vector $\mathbf{v}^{\prime \prime}$ is $(150,300,0)$. It is easy to verify that this vector is also feasible. Similarly, we obtain that $\operatorname{Profit}\left(Q^{\prime}, \mathbf{v}^{\prime \prime}\right)$ is equal to $\$ 200$.

In the above example, we learn that even though we select the same two packages (i.e., $q_{1}$ and $q_{2}$ ), different price assignment vectors give different profits. It is obvious that price assignment vector $\mathbf{v}^{\prime}$ is better than price assignment vector $\mathbf{v}^{\prime \prime}$ because it gives more profit.

Since the company wants to maximize its profit, it wants to find a price assignment vector which maximizes its profit.

Definition 3 (Optimal Price Assignment Vector): Let $Q^{\prime}$ be a set of $k$ tuples selected from $Q$. Let $\mathcal{V}$ be a set of all possible feasible price assignment vectors for $Q^{\prime}$. The optimal price assignment vector of $Q^{\prime}$ is defined to be the price assignment vector $\mathbf{v}_{o}$ for $Q^{\prime}$ such that

$$
\operatorname{Profit}\left(Q^{\prime}, \mathbf{v}_{o}\right)=\max _{\mathbf{v}^{\prime} \in \mathcal{V}} \operatorname{Profit}\left(Q^{\prime}, \mathbf{v}^{\prime}\right)
$$

The optimal profit of $Q^{\prime}$, denoted by $\operatorname{Profit}_{o}\left(Q^{\prime}\right)$, is defined to be $\operatorname{Profit}\left(Q^{\prime}, \mathbf{v}_{o}\right)$ where $\mathbf{v}_{o}$ is the optimal price assignment vector of $Q^{\prime}$. 
In Section III, we describe an efficient algorithm to find the optimal price assignment vector given a set $Q^{\prime}$ of $k$ selected tuples.

We just learnt that given a particular set $Q^{\prime}$, we can determine the optimal profit of $Q^{\prime}$. However, there are many possible subsets of $Q$ containing $k$ tuples. The company wants to find a selection containing $k$ tuples from $Q$ such that the total profit is maximized and $k$ tuples will be selected by some customers.

Problem 1 (Finding Top- $k$ Profitable Products): Let $\mathcal{Q}$ be the set of all possible subsets containing $k$ tuples from $Q$. We want to select a set $Q^{\prime}$ of $k$ tuples from $Q$ such that Profit $_{o}\left(Q^{\prime}\right)=\max _{Q^{\prime \prime} \in \mathcal{Q}} \operatorname{Profit}_{o}\left(Q^{\prime \prime}\right)$

This problem is called finding top- $k$ profitable products $(T P P)$.

A naive way for this problem is to enumerate all possible subsets of size $k$ from $Q$, calculate the optimal profit of each possible subset, and choose the subset with the greatest profit. However, this approach is not scalable because there are an exponential number of all possible subsets. This motivates us to propose efficient algorithms for problem TPP which will be described in Section IV and Section V.

\section{Finding Optimal Price Assignment}

In this section, we present an algorithm for finding the optimal price assignment called $A O P A$ in $O(k(\log (m+n)+$ $N)$ ) time given a set $Q^{\prime}$ of size $k$ where $N<<(m+n)$.

Suppose that $Q^{\prime}$ is a selection set. Our objective is to find the optimal price assignment vector of $Q^{\prime}$. After setting the prices of all tuples in $Q^{\prime}$ according to this vector, the tuples in $Q^{\prime}$ are in the skyline with respect to $P \cup Q^{\prime}$.

Let $X=P \cup Q^{\prime}$. Given $p \in X$ and $p^{\prime} \in X, p$ is said to quasi-dominate $p^{\prime}$ if (1) $p$ dominates $p^{\prime}$ with respect to the first $l-1$ attributes, namely $A_{1}, A_{2}, \ldots, A_{l-1}$, or (2) $p$ has the same $l-1$ attribute values as $p^{\prime}$. In our running example, $l=2$. Suppose that $Q^{\prime}=\left\{q_{1}, q_{2}\right\}, p_{2}$ quasi-dominates both $p_{1}$ and $q_{1}$ since $p_{2}$ dominates both $p_{1}$ and $q_{1}$ with respect to attribute "Distance-to-Beach". $p_{2}$ also quasi-dominates $q_{2}$. Let $\gamma\left(X, q_{i}\right)$ be a set containing all tuples in $X$ which quasi-dominate $q_{i}$. For example, suppose that $Q^{\prime}=\left\{q_{1}, q_{2}\right\} . \gamma\left(X, q_{1}\right)=$ $\left\{q_{2}, p_{2}, p_{3}, p_{4}\right\}$ and $\gamma\left(X, q_{2}\right)=\left\{p_{2}, p_{3}, p_{4}\right\}$.

The following lemma gives us an intuition of how to design an algorithm to find the optimal price assignment vector of $Q^{\prime}$.

Lemma 1: Suppose that $p \in X$ and $q_{i} \in Q^{\prime}$. Consider that we are given a price assignment vector of $Q^{\prime}$ equal to $\mathbf{v}=$ $\left(v_{1}, v_{2}, \ldots, v_{n}\right)$ such that we set the price of each $q_{j}$ (i.e., $\left.q_{j} . A_{l}\right)$ in $Q^{\prime}$ to $v_{j}$. If $p$ dominates $q_{i}$, then $p \in \gamma\left(X, q_{i}\right)$.

According to the above lemma, we divide the tuples in $X$ into two groups.

- Group 1 (Outside $\gamma$ ): Group 1 is the set of all tuples not in $\gamma(X, q)$ (more specifically, all tuples in $X-\gamma(X, q)$ ). The tuples in this group do not dominate $q$ regardless of any price assignment vector of $Q^{\prime}$.

In our running example, let $Q^{\prime}=\left\{q_{1}, q_{2}\right\}$. Consider $q_{1}$. Since $p_{1}$ is not in $\gamma\left(X, q_{1}\right)$, we know that $p_{1}$ does not dominate $q_{1}$.

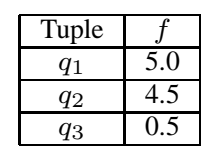

TABLE III

The $f$ VAlue of EACH TUPLE $p \in Q$

- Group 2 (Inside $\gamma$ ): Group 2 is the set of all tuples in $\gamma(X, q)$. For a particular price assignment vector of $Q^{\prime}$, some tuples in this group may dominate $q$ while for another particular price assignment vector of $Q^{\prime}$, they may not dominate $q$.

For example, consider $q_{1}$ again. Consider a price assignment vector $\mathbf{v}=(300,300,0)$. Note that $q_{2}$ is in $\gamma\left(X, q_{1}\right)$. After we set the prices of $q_{1}$ and $q_{2}$ with vector $\mathbf{v}, q_{2}$ dominates $q_{1}$.

Consider another price assignment vector $\mathbf{v}^{\prime}=$ $(250,300,0)$. After we set the prices of $q_{1}$ and $q_{2}$ with vector $\mathbf{v}^{\prime}, q_{2}$ does not dominate $q$.

Our objective is to make sure that each tuple $q \in Q^{\prime}$ is in the skyline with respect to $X\left(=P \cup Q^{\prime}\right)$. That is, each tuple $q$ in $Q^{\prime}$ is not dominated by any tuple in $X$. This is our goal. Consider Group 1 (Outside $\gamma$ ). We can achieve the goal because all tuples in this group do not dominate $q$. Consider Group 2 (Inside $\gamma$ ). It is possible that some tuples in $\gamma(X, q)$ dominate $q$ for a particular price assignment vector. For another price assignment vector, they do not dominate $q$.

In the above, we learn that if we want to determine the price of $q_{i}$ in $Q^{\prime}$ such that $q_{i}$ is in the skyline, we only need to consider the tuples in $\gamma(X, q)$.

Given a tuple $q \in Q^{\prime}$, we know that only the tuples in $X$ quasi-dominating $q$ affect the price of $q$. Note that the prices of all tuples in $P$ are given and the prices of all tuples in $Q^{\prime}$ are to be found. Thus, according to the quasi-dominance relationship, we design a progressive algorithm which finds the price of each tuple $q$ in $Q^{\prime}$ by the following principle.

Principle 1: Whenever we want to find the price of $q_{i}$ in $Q^{\prime}$, we make sure that the prices of all tuples in $Q^{\prime}$ quasidominating $q_{i}$ have already been determined.

Next, we need to determine the ordering of processing tuples in $Q^{\prime}$ which follows the above principle.

We define the following monotonically increasing function $f$ which can determine the ordering. Given a tuple $q$ in $Q$, function $f$ is defined as follows.

$$
f(q)=\sum_{i=1}^{l-1} q \cdot A_{i}
$$

In our running example, $l=2$. The $f$ value of each tuple $q \in Q$ can be found in Table III. The ordering of tuples in $X$ is $q_{3}, q_{2}$ and $q_{1}$.

With this function $f$, we know the following lemma.

Lemma 2: Suppose $p$ and $p^{\prime}$ are in $X$. If $p$ quasi-dominates $p^{\prime}$, then $f(p)$ is smaller than or equal to $f\left(p^{\prime}\right)$.

For example, since $p_{2}$ quasi-dominates $p_{1}, f\left(p_{2}\right)(=4)$ is smaller than $f\left(p_{1}\right)(=7)$. 

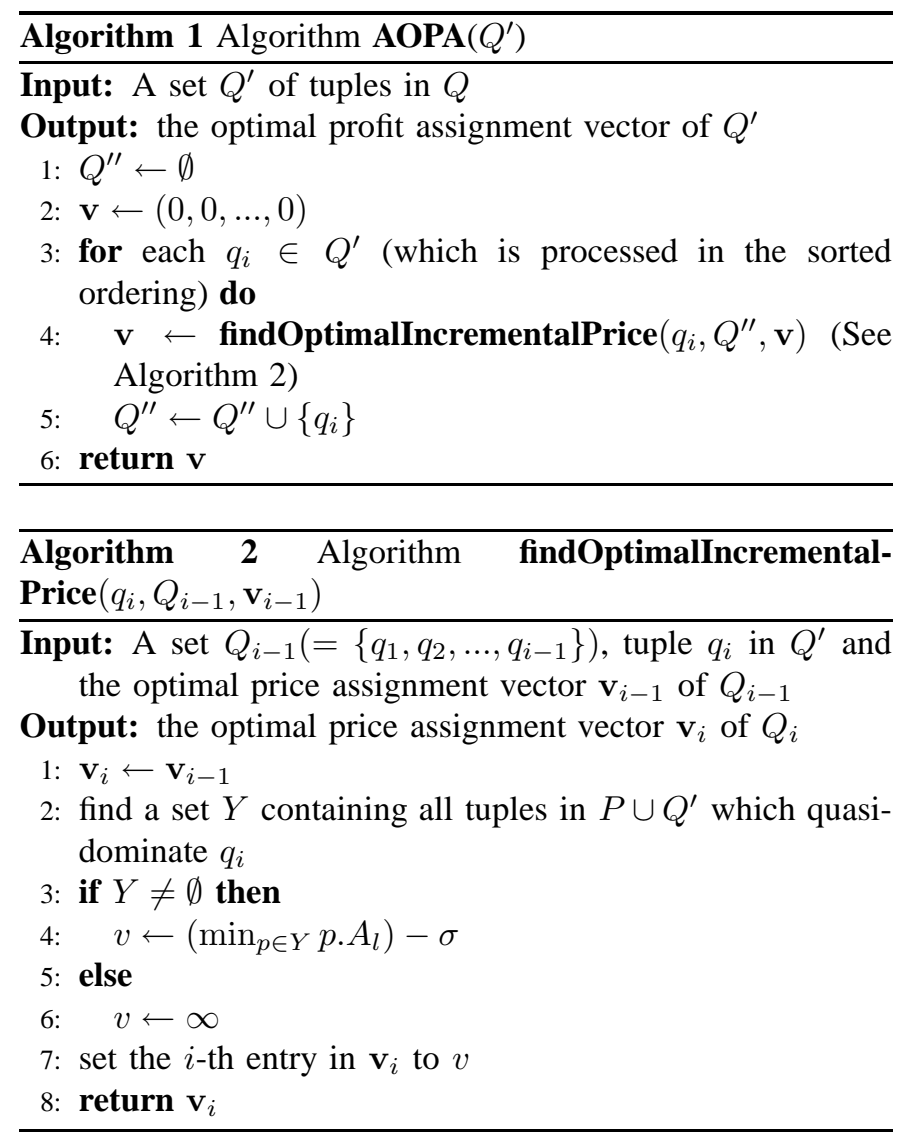

With the above lemma, we can first compute the $f$ values of all tuples in $Q^{\prime}$. We sort the tuples in $Q^{\prime}$ in ascending order of these $f$ values. Then, we determine the price of each tuple $q$ in $Q^{\prime}$ according to this ordering, which follows Principle 1.

After we obtain the ordering of processing the tuples in $Q^{\prime}$, we present an algorithm to determine the optimal price assignment vector of $Q^{\prime}$ incrementally.

Without loss of generality, we assume that $q_{1}, q_{2}, \ldots, q_{k}$ are the tuples in $Q^{\prime}$ sorted in ascending order of the $f$ values. Let $Q_{0}=\emptyset$. Let $Q_{i}=Q_{i-1} \cup\left\{q_{i}\right\}$ where $i=1,2,3, \ldots, k$.

Lemma 3: Suppose that $p \in X$ and $q_{i} \in Q^{\prime}$. Consider that we are given the optimal price assignment vector of $Q_{i-1}$ equal to $\mathbf{v}_{i-1}=\left(v_{1}, v_{2}, \ldots, v_{n}\right)$ such that we set the price of each $q_{j}$ (i.e., $\left.q_{j} . A_{l}\right)$ in $Q_{i-1}$ to $v_{j}$. Suppose that $\gamma\left(X, q_{i}\right) \neq \emptyset$. Let $\mathbf{v}_{i}$ be a price assignment vector equal to $\mathbf{v}_{i-1}$ except that the $i$-th entry of $\mathbf{v}_{i}$ is set to $\left(\min _{p \in \gamma\left(X, q_{i}\right)} p \cdot A_{l}\right)-\sigma . \mathbf{v}_{i}$ is the optimal price assignment vector of $Q_{i}$.

By Lemma 3, we can derive a progressive algorithm as shown in Algorithm 1.

With Lemma 3, it is easy to verify the following theorem.

Theorem 1: Given a set $Q^{\prime}$ of $k$ tuples selected from $Q$, Algorithm AOPA returns the optimal price assignment of $Q^{\prime}$.

Implementation and Time Complexity: In Algorithm 2, the most time-consuming operation is the step of finding all tuples in $P \cup Q^{\prime}$ which quasi-dominate $q_{i}$. One possible implementation is to build an $\mathrm{R}^{*}$-tree index on dataset $P \cup Q^{\prime}$ according to the first $l-1$ attributes. If we perform a range query with the range equal to " $A_{i} \leq q_{i}$. $A_{i}$ " for each $i \in$ $[1, l-1]$, then we can find all tuples in $P \cup Q^{\prime}$ which quasidominate $q_{i}$. However, with this implementation, we have to build different indexes on $P \cup Q^{\prime}$ for different selection sets $Q^{\prime}$, which is not efficient.

Another possible implementation is to build an $\mathrm{R}^{*}$-tree index on dataset $P \cup Q$ (instead of $P \cup Q^{\prime}$ ) according to the first $l-1$ attributes. Similarly, we perform a range query with the same range as above and find all tuples in $P \cup Q$ which quasidominate $q_{i}$. In this implementation, since $Q^{\prime} \subseteq Q$, we can do a post-processing step to select all tuples in $P \cup Q^{\prime}$ which quasi-dominate $q_{i}$. This implementation has its advantage that we only need to build an index once for any selection set. We adopt this implementation in our experiment.

Suppose that we are given an $\mathrm{R}^{*}$-tree index on dataset $P \cup Q$ in the second implementation. We want to analyze the time complexity of the step of finding all tuples in $P \cup Q^{\prime}$ which quasi-dominate $q_{i}$. In most cases, the cost of a range query is $O(\log (|P|+|Q|)+N)$ where $N$ is the total number of tuples returned in a range query. Typically, $N$ is extremely small compared with $(|P|+|Q|)$. That is, $N<<|P|+|Q|$. The post-processing step takes $O(N)$ time. Thus, the step of finding all tuples in $P \cup Q^{\prime}$ which quasi-dominate $q_{i}$ takes $O(\log (|P|+|Q|)+N)$ time.

After we analyze the time complexity of this timeconsuming operation, it is easy to verify that Algorithm 2 takes $O(\log (|P|+|Q|)+N)$ time.

Consider Algorithm 1. Since there are $\left|Q^{\prime}\right|$ iterations (in lines 3-5) and each iteration calls Algorithm 2 (which takes $O(\log (|P|+|Q|)+N)$ time), the overall time complexity of Algorithm 1 is $O\left(\left|Q^{\prime}\right|(\log (|P|+|Q|)+N)\right)$. Since $m=$ $|P|, n=|Q|$ and $k=\left|Q^{\prime}\right|$, the time complexity becomes $O(k(\log (m+n)+N))$.

\section{DYNAMic Programming}

In this section, we present a dynamic programming approach which finds an optimal solution for problem TPP when $l=2$.

Consider $l=2$. Without loss of generality, we assume that $q_{1}, q_{2}, . ., q_{n}$ are sorted in ascending order of the $f$ values. $Q(i)$ is defined to be a set of tuples in $Q$ such that these tuples are quasi-dominated by $q_{i}$. Let $S(i, t)$ denote the set $Q^{\prime}$ of size $t$ where $i \in[1, n]$ and $t \in[0, k]$ such that $\operatorname{Profit}_{o}\left(Q^{\prime}\right)=\max _{Q^{\prime \prime} \in \mathcal{Q}} \operatorname{Profit}_{o}\left(Q^{\prime \prime}\right)$ where $\mathcal{Q}$ is the set of all possible subsets containing $t$ tuples from $Q(i)$. Let $\mathbf{v}(i, t)$ denote the optimal price assignment vector of set $S(i, t)$. Let $T(i, t)$ denote the (optimal) profit of set $S(i, t)$.

Let us use a symbol $\alpha\left(q_{i}, S, \mathbf{v}\right)$ to represent findOptimalIncrementalPrice $\left(q_{i}, S, \mathbf{v}\right)$.

In the following, we describe how to find three variables, namely $\mathbf{v}(i, t), T(i, t)$ and $S(i, t)$. Consider two cases.

- Case 1: $q_{i}$ is included in the final selection of size $t$. By Lemma 3, the optimal price assignment vector of $S(i, t)$, denoted by $\mathbf{v}(i, t)$, can be obtained from the 
optimal price assignment vector of $S(i-1, t-1)$, denoted by $\mathbf{v}(i-1, t-1)$.

Thus, we have the following equation.

$$
\mathbf{v}(i, t)=\alpha\left(q_{i}, S(i-1, t-1), \mathbf{v}(i-1, t-1)\right)
$$

Thus,

$$
T(i, t)=T(i-1, t-1)+v
$$

where $v$ is the $i$-th entry in $\mathbf{v}(i, t)$. Let $T_{\text {select }}=T(i-$ $1, t-1)+v$.

Similarly, $S(i, t)$ can be obtained as follows.

$$
S(i, t)=S(i-1, t-1) \cup\left\{q_{i}\right\}
$$

- Case 2: $q_{i}$ is not included in the final selection.

We have

$$
\mathbf{v}(i, t)=\mathbf{v}(i-1, t)
$$

We have

$$
T(i, t)=T(i-1, t)
$$

and

$$
S(i, t)=S(i-1, t)
$$

Let $T_{\text {not Select }}=T(i-1, t)$.

Note that we want to maximize the profit of the selection set of size $t$. Obviously, if $T_{\text {select }} \geq T_{\text {notSelect }}$, we should select $q_{i}$ in the selection set (which corresponds to Case 1). Otherwise, we should not select $q_{i}$ (which corresponds to Case 2).

The pseudo-code of the dynamic programming approach is shown in Algorithm 3.

Theorem 2: Algorithm Dynamic Programming returns an optimal solution $Q^{\prime}$ of size $k$ for problem TPP (i.e., the set $Q^{\prime}$ of size $k$ with the greatest profit) when $l=2$.

Time Complexity: Consider Algorithm 3. Statements from line 1 to line 3 takes $O(n)$ time while statements from line 4 to line 6 takes $O(k \cdot k(\log (m+n)+N))=O\left(k^{2}(\log (m+n)+N)\right)$ time. Consider statements from line 7 to line 22. There are $O(k n)$ iterations where the statements from line 9 to line 22 correspond to an iteration. It is easy to verify that each iteration takes $O(k(\log (m+n)+N)+n)$ time. Thus, statements from line 7 to line 22 takes $O(k n(k(\log (m+n)+N)+n))=$ $O\left(k^{2} n(\log (m+n)+N)+k n^{2}\right)$ time.

\section{GReEdy Algorithm}

In the previous section, we described a dynamic programming approach which finds an optimal solution when $l=2$. However, when $l>2$, we show that the problem is NP-hard as follows.

Theorem 3: When $l>2$, problem TPP is NP-hard.

The proof can be found in the appendix.

Since the problem is NP-hard, we propose two greedy algorithms for this problem. As we described in Example 3, the price of a new selected tuple may affect the price of another

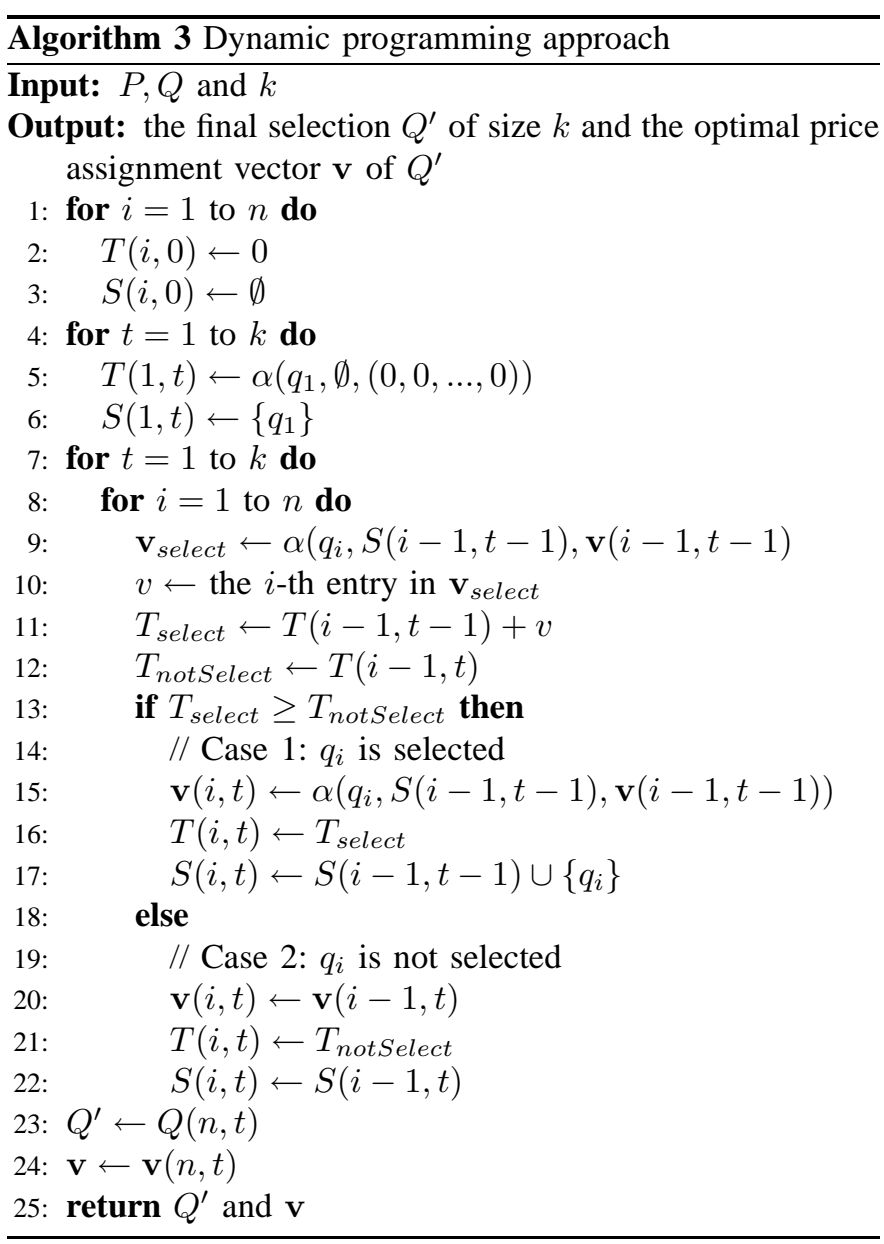

new selected tuple. We call this phenomenon a price correlation. The first version of the greedy algorithm is the algorithm which selects tuples in $Q$ iteratively without considering the price correlation. The first greedy algorithm returns a solution with a theoretical guarantee on the profit. The second version is the algorithm which selects tuples in $Q$ iteratively considering the price correlation. The second greedy algorithm performs well empirically.

\section{A. Greedy Based Algorithm I}

The first version of the greedy algorithm is the algorithm which selects tuples in $Q$ iteratively without considering the price correlation.

For each tuple $q$ in $Q$, we first define the optimal profit of the selection set containing $q$ only. We call this profit the standalone profit of $q_{i}$.

Definition 4 (Standalone Profit): Given a tuple $q$ in $Q$, the standalone profit of $q$, denoted by $S P(q)$, is defined to $\operatorname{Profit}_{o}(\{q\})$.

The first version of the greedy algorithm is described as follows. Specifically, for each tuple $q$ in $Q$, we find the standalone profit of $q$. Then, we choose $k$ tuples which have the greatest standalone profits. This version is shown in Algorithm 4. 


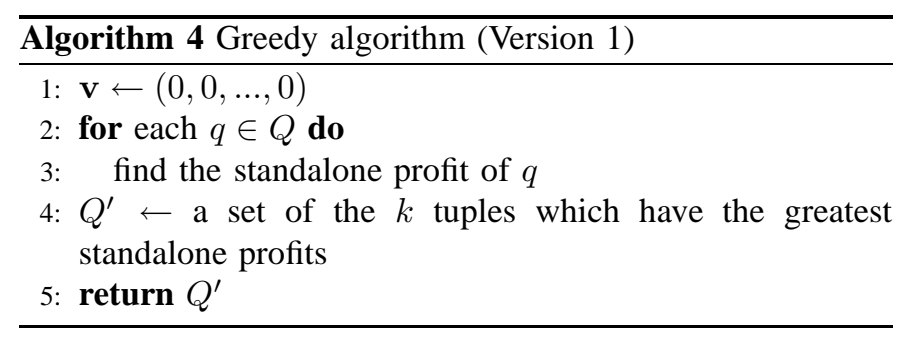

Although this greedy approach is a heuristical approach, it has theoretical guarantees on the profit returned by the algorithm.

Suppose that $O$ is the optimal selection set for problem TPP (i.e., the selection set which has the greatest profit). Note that the optimal profit of $O$ is equal to $\operatorname{Profit}_{o}(O)$. Recall that we want to maximize the profit, due to the heuristical nature of the greedy algorithm, this algorithm may return a selection $Q^{\prime}$ which has a lower profit (which is equal to $\operatorname{Profit}_{o}\left(Q^{\prime}\right)$ ). It is easy to verify that

$$
\operatorname{Profit}_{o}\left(Q^{\prime}\right) \leq \operatorname{Profit}_{o}(O)
$$

In the following, we give two theoretical results about the error guarantee on the profit returned by the algorithm. The first result corresponds to an additive error guarantee while the second one corresponds to a multiplicative error guarantee.

We first show the result about the additive error guarantee.

Theorem 4: Let $O$ be the optimal selection set and $Q^{\prime}$ be the selection set returned by Algorithm 4.

$$
\operatorname{Profit}_{o}(O)-\epsilon_{a d d} \leq \operatorname{Profit}_{o}\left(Q^{\prime}\right)
$$

where

$$
\epsilon_{a d d}=\frac{k(k-1)}{2} \sigma
$$

Next, we show the result about the multiplicative error guarantee.

Theorem 5: Let $O$ be the optimal selection set and $Q^{\prime}$ be the selection set returned by Algorithm 4. Suppose that Profit $_{o}\left(Q^{\prime}\right)>0$. Let $\Delta=\sum_{q_{i} \in Q^{\prime}} S P\left(q_{i}\right)$. Algorithm 4 is a $\left(1-\epsilon_{\text {mult }}\right)$-approximate algorithm. That is,

$$
\operatorname{Profit}_{o}\left(Q^{\prime}\right) \geq\left(1-\epsilon_{\text {mult }}\right) \operatorname{Profit}_{o}(O)
$$

where $\epsilon_{\text {mult }}=\frac{k(k-1) \sigma}{2 \Delta}$.

Time Complexity: Consider Algorithm 4. We need to calculate the standalone profit of $q$ for each tuple $q \in Q$. This step takes $O(k(\log (m+n)+N))$ time. Then, we need to choose the $k$ tuples which have the greatest standalone profits, which can be done in $O(k \log k)$ time. Thus, the time complexity of Algorithm 4 is $O(k(\log (m+n)+N)+k \log k)$. Since $k=O(m+n)$, the complexity becomes $O(k(\log (m+n)+$ $N))=O(k \log (m+n)+k N)$.

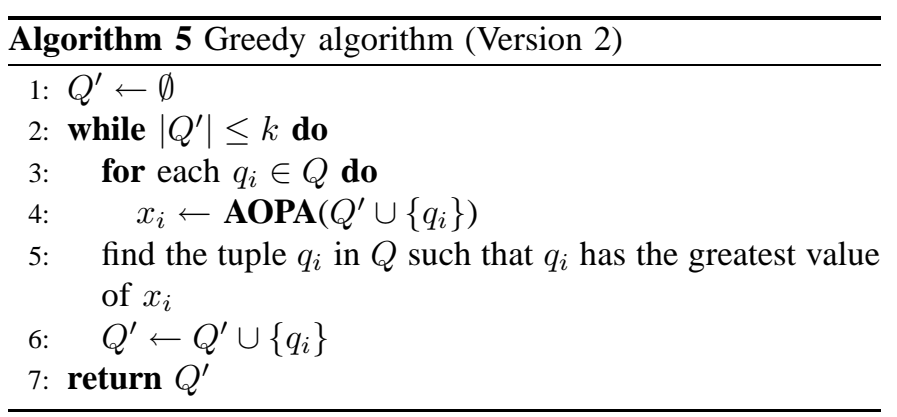

\section{B. Greedy Based Selection II}

In the previous subsection, we describe the first version of the greedy algorithm which does not consider the price correlation. In this subsection, we describe the second version of the greedy algorithm which select tuples in $Q$ iteratively considering the price correlation.

The second version of our greedy algorithms is shown in Algorithm 5.

Time Complexity: Consider Algorithm 5. There are $O(k)$ iterations where statements from line 3 to line 6 correspond to an iteration. Consider an iteration. Statements from line 3 to line 4 take $O(n \cdot k(\log (m+n)+N))=O(n k(\log (m+n)+N))$ time. Statements from line 5 to line 6 takes $O(n \log n)$ time. Thus, each iteration takes $O(n k(\log (m+n)+N)+n \log n)=$ $O(n k(\log (m+n)+N))$ time. The overall time complexity of Algorithm 5 is $O(k \cdot n k(\log (m+n)+N))=O\left(n k^{2}(\log (m+\right.$ $n)+N)$ ). Note that compared with the time complexity of Algorithm 4 (i.e., $O(k \log (m+n)+k N))$, the time complexity of Algorithm 5 is higher.

\section{DISCUSSION}

In problem TPP, after we set the price of each tuple in the selection set $Q^{\prime}$, we know that each of these tuples is in the skyline with respect to $P \cup Q^{\prime}$. In other words, after we set the price of each tuple in $Q^{\prime}$, each of these tuples is one of the best choices for the customer to choose (because there may be more than one tuple in the skyline). In order to make sure that each tuple in $Q^{\prime}$ will be chosen by a customer in the market with a higher probability, we would like to set the price of each of these tuples such that not only each of these tuples is in the skyline but also each of these tuples dominates at least $h$ tuples in the existing market $P$ where $h$ is an input parameter. This problem is called Finding top- $k$ profitable products (TPP) with the $h$-dominance constraint. The $h$-dominance constraint corresponds to that each of these tuples dominates at least $h$ tuples in the existing market $P$.

All the formulations described in Section II can also be used. The only change we need is the definition of a feasible price assignment vector. With the $h$-dominance constraint, a price assignment vector $\mathbf{v}$ is said to be feasible if after we set the price of each $q_{i} \in Q^{\prime}$ to $v_{i}$, each $q_{i} \in Q^{\prime}$ is in the skyline with respect to $P \cup Q^{\prime}$ and each $q_{i} \in Q^{\prime}$ dominates at least $h$ tuples in $P$. 


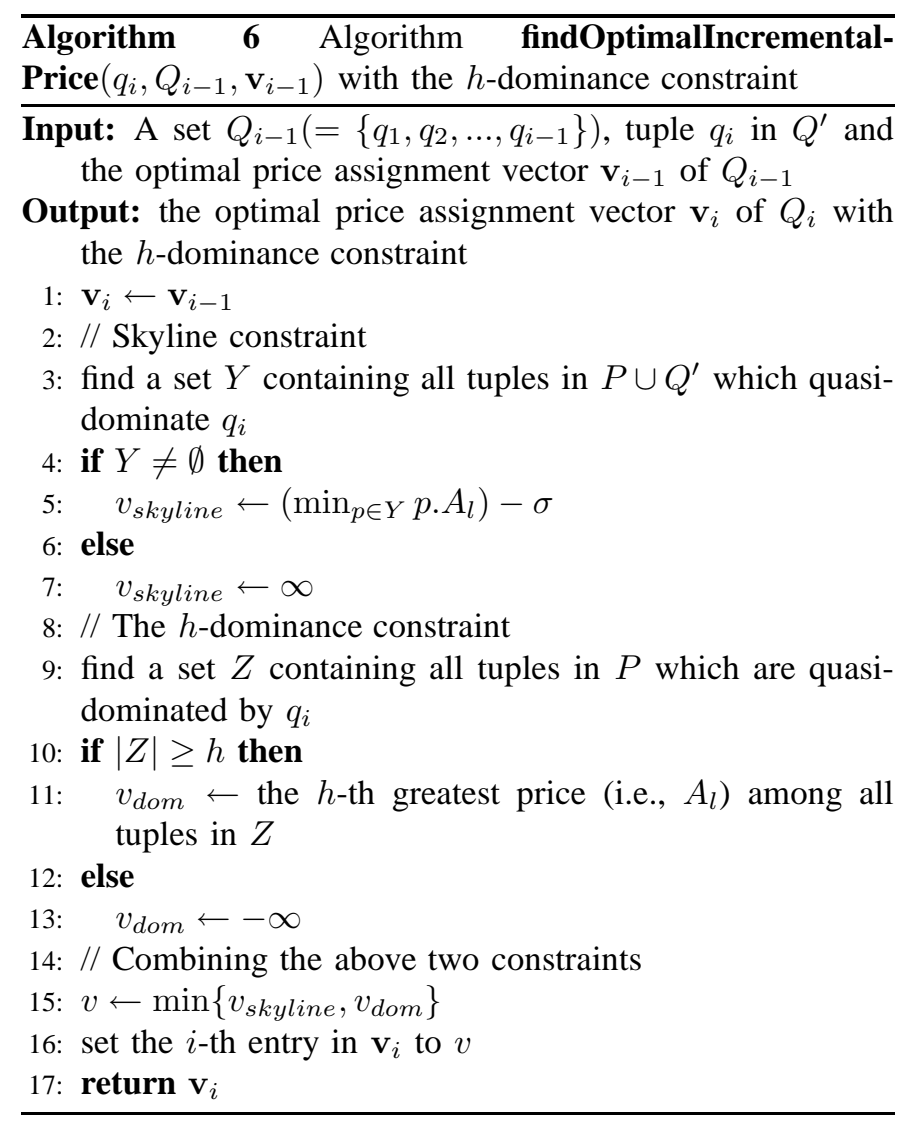

Problem TPP with the $h$-dominance constraint is more general than problem TPP without the $h$-dominance constraint. This is because if we set $h=0$, then the new problem becomes problem TPP without the $h$-dominance constraint.

After we consider the additional $h$-dominance constraint, the price assignment vector of a selection set may also be affected.

In this new problem, we only need to modify Algorithm 2 to Algorithm 6. Algorithm 6 is nearly the same as Algorithm 2. Algorithm 6 involves three major parts. The first part contains the statements related to the skyline constraint which can be found from Line 2 to Line 7 . The second part contains the statements related to the $h$-dominance constraint which can be found from Line 8 to Line 13. The third part contains the statements related to combining the above two constraints which can be found from Line 14 to Line 16.

With the following theorem (which is similar to Lemma 3), it is easy to prove the correctness of Algorithm 6 (i.e., under the $h$-dominance constraint, Algorithm 6 returns the optimal price assignment vector of $Q_{i}$ given a set $Q_{i-1}=$ $\left\{q_{1}, q_{2}, \ldots, q_{i-1}\right\}$, tuple $q_{i}$ in $Q^{\prime}$ and the optimal price assignment vector of $Q_{i-1}$ ).

Theorem 6: Suppose that $p \in X$ and $q_{i} \in Q^{\prime}$. Consider that we are given the optimal price assignment vector of $Q_{i-1}$ equal to $\mathbf{v}_{i-1}=\left(v_{1}, v_{2}, \ldots, v_{n}\right)$ such that we set the price of each $q_{j}$ (i.e., $q_{j} . A_{l}$ ) in $Q_{i-1}$ to $v_{j}$.

- Skyline Constraint: Suppose that $\gamma\left(X, q_{i}\right) \neq \emptyset$. Let $v_{\text {skyline }}=\left(\min _{p \in \gamma\left(X, q_{i}\right)} p \cdot A_{l}\right)-\sigma$.

- The $h$-dominance Constraint: Let $\theta\left(q_{i}\right)$ be the set containing all tuples in $P$ which are quasi-dominated by $q_{i}$. Suppose that $\theta\left(q_{i}\right) \neq \emptyset$. Let $v_{d o m}$ be the $h$-th greatest price (i.e., $A_{l}$ ) among all tuples in $Z$.

Let $\mathbf{v}_{i}$ be a price assignment vector equal to $\mathbf{v}_{i-1}$ except that the $i$-th entry of $\mathbf{v}_{i}$ is set to $\min \left\{v_{\text {skyline }}, v_{d o m}\right\} . \mathbf{v}_{i}$ is the optimal price assignment vector of $Q_{i}$.

Note that this problem with the $h$-dominance constraint is a general problem of problem TPP. Since problem TPP is NPhard, this problem is also NP-hard.

Theorem 7: The problem with the $h$-dominance constraint is NP-hard.

All the proposed algorithms, namely the dynamic approach and the two version of the greedy approach, can also be adopted except that we need to call a new version of algorithm findOptimalIncrementalPrice (Algorithm 6). Details can be found in [13].

\section{EMPIRICAL STUDIES}

We have conducted extensive experiments on a Pentium IV $2.4 \mathrm{GHz}$ PC with 4GB memory, on a Linux platform. We implemented all algorithms we proposed, namely $D P$, $G R 1$ and GR2. DP corresponds to our dynamic programming approach while $G R 1$ and $G R 2$ correspond to the first version and the second version of the greedy algorithms. We also implemented a naive (or brute-force) algorithm described in Section II. We name it as $B F$. All the program are implemented in $\mathrm{C}++$. In the following, we consider problem TPP with the $h$ dominance constraint discussed in Section VI since it is more general than problem TPP without the $h$-dominance constraint.

We measured the algorithms with four measurements, namely (1) Execution Time, (2) Preprocessing Time, (3) Memory Cost and (4) Profit. (1) The execution time of an algorithm corresponds to the time it takes to find the final selection. (2) The preprocessing of an algorithm corresponds to the time it builds a $\mathrm{R}^{*}$-tree index for quasi-dominance checking. (3) The memory cost of an algorithm is the memory occupied by the algorithm. (4) The profit of an algorithm corresponds to the profit returned by the algorithm.

\section{A. Dataset Description}

The experiments are conducted over real datasets and synthetic datasets.

1) Real Dataset: For the real datasets, same as [12], we obtain real datasets from Priceline.com and Expedia.com.

For the website of Priceline.com, we obtained all packages on Jan 15, 2009 for a round trip traveling from San Francisco to New York for a period from March 1, 2009 to March 7, 2009. We have 149 packages. These packages form the set $P$ of existing tuples. Each package has 6 attributes, namely quality-of-room, customer-hotel-grading, hotel-class, hotel-price, class-of-flight, no-of-stops and price.

For the website of Expedia.com, we obtained all flights and all hotels on the same day (i.e., Jan 15, 2009) for the 
same round trip with the same travel period. We have 1014 hotels and 4394 flights. According to these hotels and these flights, we adopt the method proposed by [12] to generate all competitive packages. Details can be found in [12]. These competitive products form set $Q$. In this dataset, we have 4787 competitive packages. Similarly, each package in $Q$ has 6 attributes (including attribute price). Note that each package in $Q$ is associated with an additional cost attribute. In order to generate the cost attribute, for each package $q$ in this package set, we set $q . C$ to be the price of this package multiplied by a discount rate $d$ where $d$ is a user parameter. Note that although there are values in attribute price in this set $Q$, we discard all these values in the dataset because our problem is to find these values.

2) Synthetic Dataset: For synthetic datasets, we adapt the dataset generator of [1]. We observe from the real dataset that some attributes have large cardinalities but some have small cardinalities. For example, in the real dataset, price may have thousands of possible values, but no-of-stops can have only 2 or 3 possible values. We divide the attributes into two groups of nearly equal size. Note that $P$ has $l$ attributes only while $Q$ has an additional attribute $C$ in addition to the $l$ attributes. The first group contains the first half of attributes (or more specifically, $A_{1}, \ldots A_{\lfloor l / 2\rfloor}$ ) each of which has the cardinality of 10 . The second group contains the second half of attributes (or more specifically, $A_{\lfloor l / 2\rfloor+1}, \ldots, A_{l}$ ) and attribute $C$ where each attribute in this group has the cardinality of $10 \mathrm{k}$.

We generate $P$ and $Q$ in the same way except that generating $P$ involves $l$ attributes but generating $Q$ involves the first $l-1$ attributes and attribute $C$. Note that attribute $A_{l}$ of $Q$ is not considered because in our problem definition, attribute $A_{l}$ is to be found. The dataset generation process is described as follows. Firstly, we used the dataset generator provided by [1] to generate an anti-correlated dataset where each attribute value is a real number in a range between 0.0 and 1.0. Secondly, we perform a postprocessing step so that each attribute in the first group has the cardinality of 10 and each attribute in the second group has the cardinality of $10 \mathrm{k}$. For an attribute in the first group, it can be easily done by multiplying a value in this attribute by 10 and rounding it to be an integer. We can also do a similar step for an attribute in the second group.

\section{B. Result over Small Synethetic Dataset}

It is known that a dynamic programming approach is not scalable to large datasets. Besides, this dynamic programming approach solves problem TPP when $l=2$. In this section, we conducted some experiments to compare all proposed algorithms over a small two-dimensional synthetic dataset where $|P|=10,000$ and $|Q|=10,000$. We set the default parameters as $h=5, d=0.5$ and $\sigma=200$.

We vary $k$ to study the performance of the proposed algorithms. Figures 1, 2 and 3 are the results for execution time, profit and the memory cost of each algorithm, respectively.

In Figure 1, the execution time of $B F$ is very large and is very unscalable. Note the PREP is the preprocessing time

\begin{tabular}{|c|c|}
\hline Parameter & Values \\
\hline$|P|$ & $0.5 \mathrm{M}, \mathbf{1 M}, 1.5 \mathrm{M}, 2.0 \mathrm{M}$ \\
\hline$|Q|$ & $0.5 \mathrm{M}, 1 \mathrm{M} 2 \mathrm{M}, 3 \mathrm{M}$ \\
\hline$d$ & $0.25, \mathbf{0 . 5}, 0.75,1$ \\
\hline$l$ & $2, \mathbf{5}, 10,15,20$ \\
\hline$k$ & $10, \mathbf{2 0}, 50,100$ \\
\hline$\sigma$ & $50, \mathbf{1 0 0}, 150,200$ \\
\hline$h$ & $0, \mathbf{1 0}, 20,30$ \\
\hline
\end{tabular}

TABLE IV

EXPERIMENTAL SETTING

of $G R 1$ and $G R 2$. It is nearly equal to the time for $G R 1$ and $G R 2$ to find the selection set for problem TPP. In Figure 2, the profit of $D P$ and $B F$ is the greatest but the profit of $G R I$ and $G R 2$ is also high. In most cases, $G R 2$ returns higher profit than GRI. In Figure 3, as expected, DP occupies much more memory than other approaches.

Since $B F$ is not scalable, in the following, we do not compare all algorithms with $B F$.

\section{Result over Large Synthetic Dataset}

In the previous section, we conducted experiments over small synthetic datasets. In these experiments, although GRI and GR2 are heuristical, they also give a high profit. In this section, we conducted experiments over large synthetic datasets to study the scalability of GRI and GR2. We varied $|P|,|Q|, d, l, k, \sigma$ and $h$ in our experiments. The values of each parameter used in the experiments are given in Table IV where the default values are in bold.

Figures 4, 5 and 6 shows some selected results. For the sake of space, we do not show some figures.

1) Execution time: Figures (a) show the measurement of execution time. In all figures, $G R 2$ runs slower than GR1. As we discussed in Section V, the time complexity of $G R 2$ is higher than that of GRI. For factor $k$ (Figure 5(a), when $k$ increases, the execution time of $G R 2$ increases exponentially but the execution time of GRI does not change much. This is because the time complexity of $G R 2$ is quadratic with respect to $k$ but the time complexity of GRl is not.

2) Preprocessing time: Figures (b) show the preprocessing time of the algorithms. This involves the step of building the index. When $|P|,|Q|$ and $l$ increase, the preprocessing times of $G R 1$ and $G R 2$ increase.

3) Memory cost: Figures (c) show the memory cost of the algorithms. Since the memory cost of both GRI and GR2 is the memory occupied by the spatial index $\mathrm{R}^{*}$-tree on dataset $P \cup Q$, when $|Q|$ increases and $|P|$ increases, the memory cost increases, as shown in Figures 4.

4) Profit: Figures (d) show the profit returned by the algorithms. In most cases, $G R 1$ and $G R 2$ gives similar profits. For factor $k$ (Figure 5(d)), when $k$ increases, the profits of $G R 1$ and GR2 increase because more tuples are selected to contribute the profit of the final selection. For factor $h$ (Figure 6(d)), when $h$ increases, the profits of both algorithms decreases. This is because if $h$ is larger, then the price of each selected tuple in the final selection should be set lower in order that each of tuples dominates at least $h$ tuples in the existing market. 

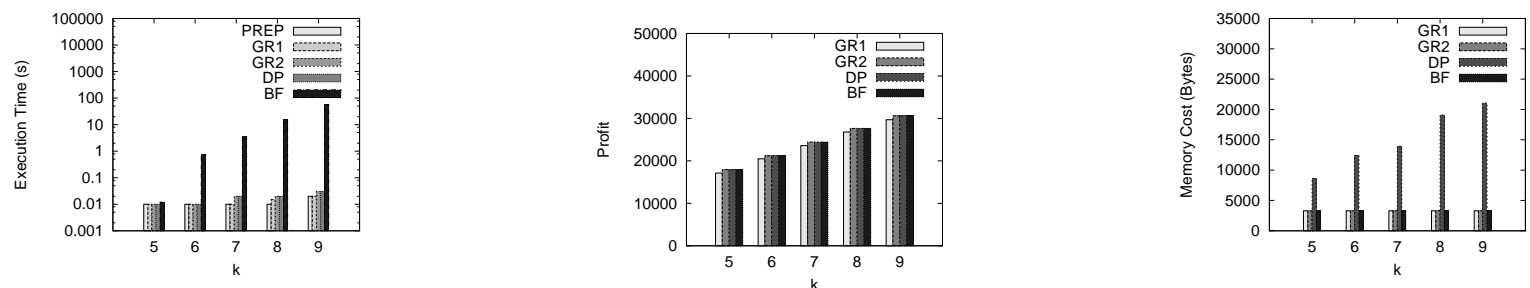

Fig. 1. Execution time of all algorithms (small dataset)

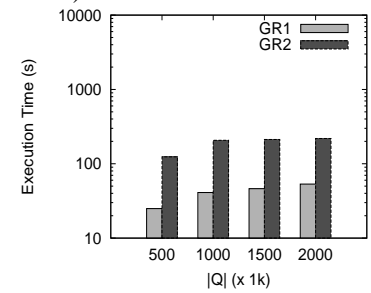

(a)

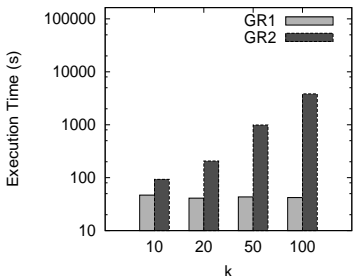

(a)

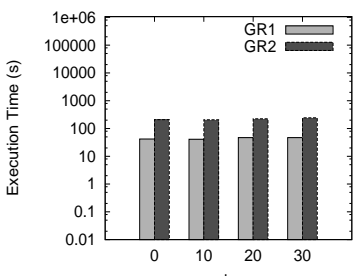

(a)

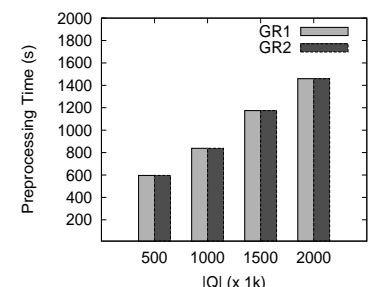

(b)

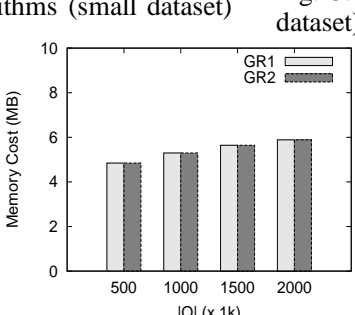

(c) dataset)

Fig. 3. Memory cost of all algorithms (small

Fig. 4. Effect of $|Q|$ (the number of potential new tuples)

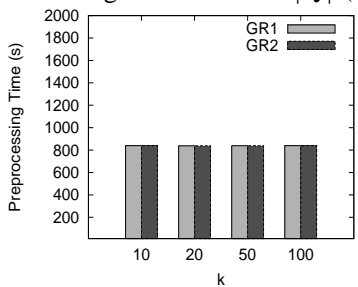

(b)

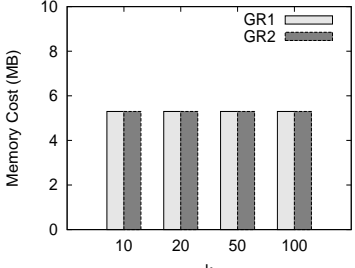

(c)

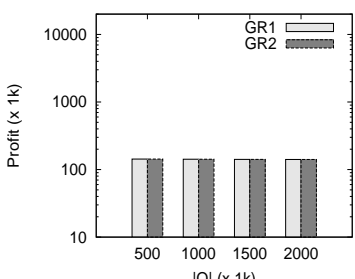

(d)

Fig. 5. Effect of $k$ (the size of the final section set)

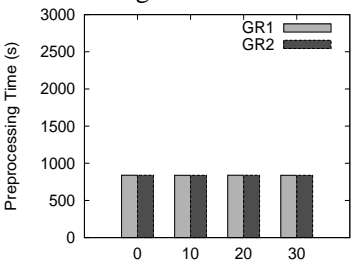

(b)

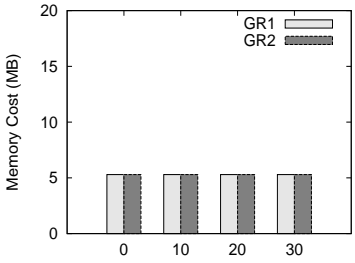

(c)

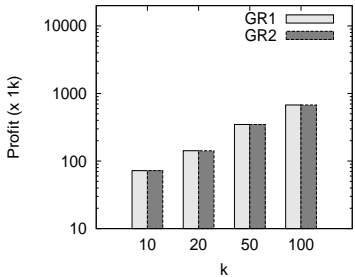

(d)

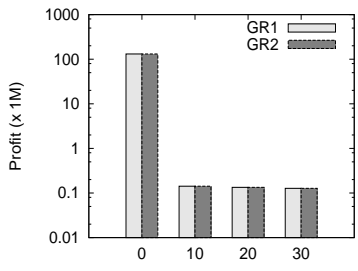

(d)

Fig. 6. Effect of $h$ (the minimum number of tuples dominated by each tuple in the selection set)

\begin{tabular}{|l|l|}
\hline$h$ & $15, \mathbf{2 0}, 25,30$ \\
\hline$\sigma$ & $1000,2000, \mathbf{5 0 0 0}, 10000$ \\
\hline$d$ & $0.4, \mathbf{0 . 6}, 0.8,1.0$ \\
\hline$k$ & $100, \mathbf{1 5 0}, 200,250$ \\
\hline
\end{tabular}

TABLE V

EXPERIMENT PARAMETERS ON REAL DATASET

\section{Result over Real Dataset}

We also conducted experiments on real datasets. We varied four factors, namely $h, k, d$ and $\sigma$. For the interest of space, we only show the results with two factors $h$ and $k$ as shown in Figures 7 and 8 , respectively. The default setting configuration is: $k=150, h=20, d=0.6$ and $\sigma=50$. The results for real datasets are similar to those for synthetic datasets.

Summary: Although $D P$ finds the optimal solution for problem TPP, it is not scalable and is limited to problem TPP when $l=2 . G R 1$ and $G R 2$ is scalable to large datasets. It is shown that they can find a selection set $Q^{\prime}$ with high profits. In most cases, GR1 and GR2 returns similar profits. However, GR2

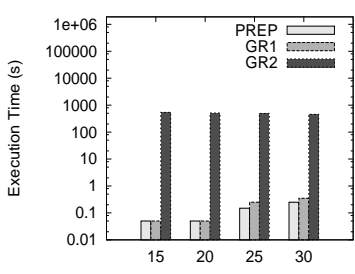

(a)

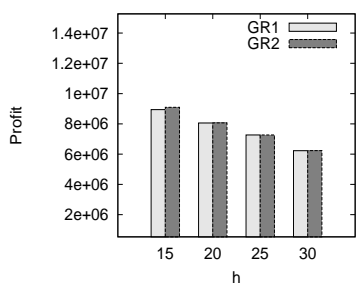

(b)
Fig. 7. Effect of $h$ (the minimum number of tuples dominated by each tuple in the selection set)

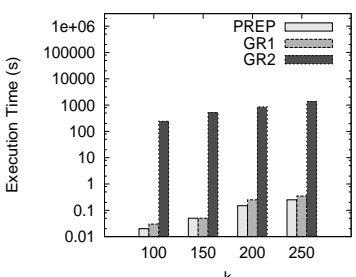

(a)

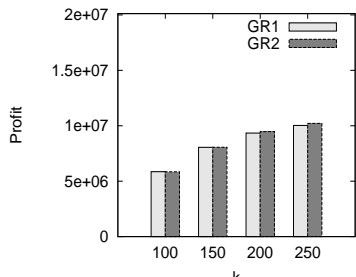

(b)
Fig. 8. Effect of $k$ (the size of the final selection set) 
sometimes gives a higher profit than GRl.

\section{RELATED WORK}

Skyline queries have been studied since 1960s in the theory field where skyline points are known as Pareto sets and admissible points [4] or maximal vectors [2]. However, earlier algorithms such as [2], [3] are inefficient when there are many data points in a high dimensional space. Skyline queries in database was first studied by Borzsonyi [1] in 2001.

After that, a lot of techniques were proposed to accelerate the computation of skyline and its variations. Here, we briefly summarize some of them. Some representative methods include a bitmap method [10], a nearest neighbor (NN) algorithm [6], and branch and bound skylines (BBS) method [8].

Top- $K$ queries about skyline were studied in [8], [7], [11]. [8] discussed ranked skyline and $K$-dominating queries. Given a set of points in $d$-dimensional space, ranked skyline specifies a monotone ranking function, and returns $k$ tuples in the $d$ dimensional space which have the smallest (or greatest) scores according to an input function. Given a set of points in $d$ dimensional space, $K$-dominating queries retrieve $K$ points that dominate the greatest number of points. It is similar to the $h$-dominance constraint introduced in Section VI.

[7], [11] studied representative skyline queries. The problem is to select $k$ points among all skyline points according to a pre-defined objective function. The $k$ points in the output are said to be representative.

[7] was the first to introduce representative skyline queries. [7] finds a set of $k$ points among all skyline points such that the number of points dominated by this set is maximized. However, the method in [7] cannot be applied in our problem because we consider both the profitability of products and the dominance relation of products, but [7] considers the dominance relation only. Besides, the price of each product is to be found in our problem.

Another definition of representative skyline queries was proposed by [11]. In [11], representative skyline queries is to find $k$ points (or $k$ representative points) among all skyline points such that the sum of the distances between each skyline point and its "closest" representative point is minimized.

All of the above studies are to find $k$ points or tuples given a single table where all attribute values of each tuple in the table are given. This paper has the following differences. Firstly, we want to find $k$ tuples given two tables (one is $P$ and the other is $Q$ ) where one of the attribute values of each tuple in one table $(Q)$ is not given and is to be found. Secondly, the concept of profits is considered in this paper but not in the above studies.

The most closely related work is [12]. Given a set $P$ of existing tuples and a number of source tables, [12] finds all tuples "generated" from the source tables such that these tuples are in the skyline with respect to the tuples in the existing market. Those tuples are called competitive tuples or products. Note that the set of all competitive tuples generated in [12] can be regarded as set $Q$ described in this paper. However, in [12], too many competitive products are generated. In their experimental studies, there are 10,000 competitive products in a real dataset. In most cases, it is good to choose some of the competitive products instead of all competitive products for promotion. One criterion is to maximize the total profit of the selection set which is studied in this paper.

\section{COnClusion}

In this paper, we identify and tackle the problem of finding top- $k$ profitable products, which has not been studied before. We propose methods to find top- $k$ profitable products efficiently. An extensive performance study using both synthetic and real datasets is reported to verify its effectiveness and efficiency. As future work, finding top- $k$ profitable products with dynamic data and finding top- $k$ profitable products with additional constraints (e.g., representative properties [7], [11]) are interesting topics.

Acknowledgements: The research is supported by HKRGC GRF 621309 and Direct Allocation Grant DAG11EG05G.

\section{REFERENCES}

[1] S. Borzsonyi, D. Kossmann, and K. Stocker. The skyline operator. In ICDE, 2001

[2] J. L. B. et al. On the average number of maxima in a set of vectors and applications. In Journal of ACM, 25(4), 1978.

[3] J. L. B. et al. Fast linear expected-time algorithms for computing maxima and convex hulls. In SODA, 1990.

[4] O. B.-N. et al. On the distribution of the number of admissable points in a vector random sample. In Theory of Probability and its Application, 11(2), 1966

[5] B. Jiang, J. Pei, X. Lin, D. W.-L. Cheung, and J. Han. Mining preferences from superior and inferior examples. In SIGKDD, 2008.

[6] D. Kossmann, F. Ramsak, and S. Rost. Shooting stars in the sky: An online algorithm for skyline queries. In $V L D B, 2002$.

[7] X. Lin, Y. Yuan, Q. Zhang, and Y. Zhang. Selecting stars: the k most representative skyline operator. In in ICDE, 2007.

[8] D. Papadias, Y. Tao, G. Fu, and B. Seeger. Progressive skyline computation in database systems. In ACM Transactions on Database Systems, Vol. 30, No. 1, 2005.

[9] D. Sacharidis, S. Papadopoulos, and D. Papadias. Topologically-sorted skylines for partially-ordered domains. In ICDE, 2009.

[10] K.-L. Tan, P. Eng, and B. Ooi. Efficient progressive skyline computation. In $V L D B, 2001$

[11] Y. Tao, L. Ding, X. Lin, and J. Pei. Distance-based representative skyline. In ICDE '09: Proceedings of the 2009 IEEE International Conference on Data Engineering, pages 892-903, Washington, DC, USA, 2009. IEEE Computer Society.

[12] Q. Wan, R. C.-W. Wong, I. F. Ilyas, M. T. Ozsu, and Y. Peng. Creating competitive products. In $V L D B, 2009$.

[13] Q. Wan, R. C.-W. Wong, and Y. Peng. Creating top-k profitable products (technical report). In http://www.cse.ust.hk/ raywong/paper/createTopKProfitableProducttechnical.pdf, 2010.

[14] R. C.-W. Wong, J. Pei, A. W.-C. Fu, and K. Wang. Mining favorable facets. In SIGKDD, 2007.

[15] Z. Zhang, L. Lakshmanan, and A. K. Tung. On domination game analysis for microeconomic data mining. In $T K D D$, to appear.

\section{APPENDIX}

\section{A. Proof of Lemmas/Theorems}

Proof of Lemma 1: If $p$ dominates $q_{i}$, and $p \notin \gamma\left(X, q_{i}\right)$, then $p$ does not quasi-dominate $q_{i}$. So, there exists at least one attribute on which $q_{i}$ is better than $p$ according to the quasidominance definition. This conflicts with our premise that $p$ dominates $q_{i}$. 
Proof of Lemma 2: If $p$ quasi-dominates $p^{\prime}$, according to the definition of $f$, this indicates that (1) $p$ dominates $p^{\prime}$ with respect to the first $l-1$ attributes, namely $A_{1}, A_{2}, \ldots, A_{l-1}$, or (2) $p$ has the same $l-1$ attribute values as $p^{\prime}$. In case 1 , obviously, $f(p)<f\left(p^{\prime}\right)$. In case $2, f(p)=f\left(p^{\prime}\right)$. Thus, $f(p) \leq f\left(p^{\prime}\right)$

Proof of Lemma 3: Suppose that we have a better assignment vector $\mathbf{v}^{\prime}$ of $Q_{i}$ in form of $\left(v_{1}^{\prime}, v_{2}^{\prime}, \ldots v_{n}^{\prime}\right)$ such that $\operatorname{Profit}\left(Q_{i}, \mathbf{v}^{\prime}\right)>\operatorname{Profit}\left(Q_{i}, \mathbf{v}_{i}\right)$.

Consider two cases. Case 1: $v_{i}^{\prime} \leq v_{i}$. In this case, $v_{i}^{\prime}-$ $q_{i} . C \leq v_{i}-q_{i} . C$. In other words, $\triangle\left(q_{i}, v_{i}^{\prime}\right) \leq \triangle\left(q_{i}, v_{i}\right)$. In addition, we know that $\mathbf{v}_{i-1}$ is the optimal price assignment vector of $Q_{i-1}$, which means $\operatorname{Profit}\left(Q_{i-1}, \mathbf{v}^{\prime}\right) \leq$ $\operatorname{Profit}\left(Q_{i-1}, \mathbf{v}_{i-1}\right)$. Thus, we have

$$
\begin{aligned}
\operatorname{Profit}\left(Q_{i}, \mathbf{v}^{\prime}\right) & =\operatorname{Profit}\left(Q_{i-1}, \mathbf{v}^{\prime}\right)+\triangle\left(q_{i}, v_{i}^{\prime}\right) \\
& \leq \operatorname{Profit}\left(Q_{i-1}, \mathbf{v}_{i-1}\right)+\triangle\left(q_{i}, v_{i}\right) \\
& =\operatorname{Profit}\left(Q_{i}, \mathbf{v}_{i}\right)
\end{aligned}
$$

Thus, Profit $\left(Q_{i}, \mathbf{v}^{\prime}\right) \leq \operatorname{Profit}\left(Q_{i}, \mathbf{v}_{i}\right)$. This leads to a contradiction.

Case 2: $v_{i}^{\prime}>v_{i}$. It is easy to verify that $\mathbf{v}^{\prime}$ is not a feasible price assignment vector. For the sake of space, we do not include the details.

Proof of Theorem 4: Let $P$ be the set of existing tuples, and $Q$ be the set of the newly created tuples. Let $O$ be the optimal selection and $Q^{\prime}$ be the selection returned by Algorithm 4 . Given $Q^{\prime} \subseteq Q$ and $q_{i} \in Q^{\prime}$, we define $\Delta_{o}\left(q_{i}, Q^{\prime}\right)=\Delta\left(q_{i}, v_{i}\right)$ where $v_{i}$ is the $i$-th entry of the optimal price assignment vector $\mathbf{v}$ of $Q^{\prime}$. Suppose $O=\left\{o_{1}, o_{2}, \ldots o_{k}\right\}$ and $Q^{\prime}=$ $\left\{q_{1}, q_{2}, \ldots q_{k}\right\}$, where $o_{i}$ and $q_{i}$ are sorted in ascending order of the $f$ values described in Section III.

Let $n_{i}$ be the greatest possible number of tuples in $Q^{\prime}$ quasidominating $q_{i}$. It is easy to verify that $\Delta_{o}\left(q_{i}, Q^{\prime}\right) \geq S P\left(q_{i}\right)-$ $n_{i} \sigma$. Note that $\sum_{i=1}^{k} n_{i} \leq \frac{k(k-1)}{2}$. We derive that

$$
\begin{aligned}
\sum_{q_{i} \in Q^{\prime}} \Delta_{o}\left(q_{i}, Q^{\prime}\right) & \geq \sum_{q_{i} \in Q^{\prime}}\left(S P\left(q_{i}\right)-n_{i} \sigma\right) \\
& =\sum_{q_{i} \in Q^{\prime}} S P\left(q_{i}\right)-\sum_{q_{i} \in Q^{\prime}} n_{i} \sigma \\
& \geq \sum_{q_{i} \in Q^{\prime}} S P\left(q_{i}\right)-\frac{k(k-1)}{2} \sigma
\end{aligned}
$$

Thus, we conclude that

$$
\sum_{q_{i} \in Q^{\prime}} \Delta_{o}\left(q_{i}, Q^{\prime}\right) \geq \sum_{q_{i} \in Q^{\prime}} S P\left(q_{i}\right)-\frac{k(k-1)}{2} \sigma
$$

Note that

$$
\begin{aligned}
\sum_{o_{i} \in O} \Delta_{o}\left(o_{i}, O\right) & \leq \sum_{o_{i} \in O} S P\left(o_{i}\right) \\
& \leq \sum_{q_{i} \in Q^{\prime}} S P\left(q_{i}\right)
\end{aligned}
$$

Therefore, we have

$$
\begin{aligned}
\operatorname{Profit}_{o}(O)-\epsilon_{\text {add }} & =\sum_{o_{i} \in O} \Delta_{o}\left(o_{i}, O\right)-\frac{k(k-1)}{2} \sigma \\
& \leq \sum_{q_{i} \in Q^{\prime}} S P\left(q_{i}\right)-\frac{k(k-1)}{2} \sigma \\
& \leq \sum_{q_{i} \in Q^{\prime}} \Delta_{o}\left(q_{i}, Q^{\prime}\right) \\
& =\operatorname{Profit}_{o}\left(Q^{\prime}\right)
\end{aligned}
$$

Proof of Theorem 5: According to Theorem 4, we have that $\Delta-\frac{k(k-1)}{2} \sigma \leq \operatorname{Profit}_{o}\left(Q^{\prime}\right)$. By the nature of optimality, we also have $\operatorname{Profit}_{o}\left(Q^{\prime}\right) \leq \operatorname{Profit}_{o}(O)$. For any tuple $q \in Q^{\prime}$, the real profit cannot be larger than the standalone profit. Thus, $\Delta-\frac{k(k-1)}{2} \sigma \leq$ Profit $_{o}\left(Q^{\prime}\right) \leq \operatorname{Profit}_{o}(O) \leq \Delta$.

Therefore, if Profit $_{o}\left(Q^{\prime}\right)>0$, we have

$$
\begin{aligned}
\frac{\operatorname{Profit}_{o}\left(Q^{\prime}\right)}{\operatorname{Profit}_{o}(O)} & \geq \frac{\Delta-\frac{k(k-1) \sigma}{2}}{\Delta} \\
& =1-\frac{k(k-1) \sigma}{2 \Delta}
\end{aligned}
$$

Therefore,

$$
\operatorname{Profit}_{o}\left(Q^{\prime}\right) \geq\left(1-\epsilon_{\text {mult }}\right) \operatorname{Profit}_{o}(O)
$$

Proof of Theorem 6: It is very easy to verify that $\mathbf{v}_{i}$ is a feasible assignment vector, so we only prove the optimality. Suppose that $\mathbf{v}_{i}=\left(v_{1}, v_{2} \ldots v_{n}\right)$ is not optimal. Let $\mathbf{u}$ : $\left(u_{1} \ldots u_{n}\right)$ be another feasible price assignment vector such that $\operatorname{Profit}\left(Q_{i}, \mathbf{u}\right)>\operatorname{Profit}\left(Q_{i}, \mathbf{v}_{i}\right)$. Since $q_{1} \ldots q_{n}$ are sorted in ascending order of $f(q)$, we have $\operatorname{Profit}\left(Q_{i}, \mathbf{v}_{i}\right)=$ $\operatorname{Profit}\left(Q_{i-1}, \mathbf{v}_{i-1}\right)+\Delta\left(q_{i}, v_{i}\right)$.

Consider two cases. Case 1: $u_{i} \leq v_{i}$. In this case, $\Delta\left(q_{i}, u_{i}\right) \leq \Delta\left(q_{i}, v_{i}\right)$. Since $\mathbf{v}_{i-1}$ is the optimal price assignment vector of $Q_{i-1}$, thus $\operatorname{Profit}\left(Q_{i-1}, \mathbf{v}_{i-1}\right) \geq$ $\operatorname{Profit}\left(Q_{i-1}, \mathbf{u}\right)$. Therefore, we have

$$
\begin{aligned}
\operatorname{Profit}\left(Q_{i}, \mathbf{v}_{i}\right) & =\operatorname{Profit}\left(Q_{i-1}, \mathbf{v}_{i-1}\right)+\Delta\left(q_{i}, v_{i}\right) \\
& \geq \operatorname{Profit}\left(Q_{i-1}, \mathbf{u}\right)+\Delta\left(q_{i}, u_{i}\right) \\
& =\operatorname{Profit}\left(Q_{i}, \mathbf{u}\right)
\end{aligned}
$$

which conflicts with our previous assumption.

Case 2: $u_{i}>v_{i}$. In this case, since $v_{i}=$ $\min \left\{v_{\text {skyline }}, v_{\text {dom }}\right\}, v_{i}=v_{\text {skyline }}$ or $v_{i}=v_{\text {dom }}$. For any one of two cases, if $u_{i}>v_{i}=v_{\text {skyline }}$ or $u_{i}>v_{i}=v_{\text {dom }}$, we can immediately verify that $\mathbf{u}$ breaks the skyline constraint or the h-dominance constraint. 